\title{
NONNORMAL DIRICHLET QUOTIENTS AND NONNORMAL BLASCHKE QUOTIENTS
}

\author{
SHINII YAMASHITA
}

\begin{abstract}
ABSTRACr. There exists a nonnormal meromorphic function $f_{1} / f_{2}$ in $U=\{|z|<$ 1 \}, where $f_{1}$ and $f_{2}$ both are holomorphic functions with finite Dirichlet integrals in $U$. For each $0<\alpha<1$, there exists a nonnormal meromorphic function $B_{1} / B_{2}$ in $U$, where $B_{1}$ and $B_{2}$ both are Blaschke products with finite $\alpha$-weighted Dirichlet integrals in $U$.
\end{abstract}

1. Introduction. Let $D$ be the family of functions $f$ holomorphic in $U=\{|z|<1\}$ with finite Dirichlet integral

$$
\iint_{U}\left|f^{\prime}(z)\right|^{2} d x d y<\infty \quad(z=x+i y) .
$$

Then, a meromorphic function $g$ of the form $g=f_{1} / f_{2}$, where $f_{j} \in D, j=1,2$, is called a Dirichlet quotient in $U$. Note that each $f \in D$ is normal in $U$ in the sense of $\mathrm{O}$. Lehto and K. I. Virtanen [5]. The question then arises as to whether the quotient of two such normal functions is again normal in $U$.

\section{THEOREM 1. There exists a nonnormal Dirichlet quotient in $U$.}

Theorem 1 was claimed by N. Yanagihara [8]. However, as is pointed out by D. C. Rung in Mathematical Reviews 36 (1968), \# 1622, Yanagihara's “proof” is unfortunately a mixture of confusion and inaccuracy. We present a new proof which we believe is more comprehensible than Yanagihara's.

Let $D_{\alpha}(0<\alpha \leqslant 1)$ be the family of functions $f$ holomorphic in $U$ with finite weighted Dirichlet integral $\iint_{U}(1-|z|)^{\alpha}\left|f^{\prime}(z)\right|^{2} d x d y<\infty$. A quotient $B_{1} / B_{2}$ of two infinite Blaschke products $B_{1}$ and $B_{2}$ with no common zero is called a Blaschke quotient in $U$. Our next result is

THEOREM 2. For each $0<\alpha<1$, there exists a nonnormal Blaschke quotient $B_{1} / B_{2}$ in $U$ such that $B_{j} \in D_{\alpha}, j=1,2$.

It is easy merely to construct a nonnormal Blaschke quotient. The existence of the quotient with an additional property is noted by J. A. Cima [2, Theorem 4]; see the remark at the end of $\$ 3$.

Here we note that $D=D_{0} \subset D_{\alpha} \subset D_{\beta} \subset D_{1}=H^{2}$ for $0<\alpha<\beta<1$, where $H^{2}$ is the Hardy class in $U$ [3]. Furthermore, $f(z)=\sum_{n=0}^{\infty} a_{n} z^{n}, z \in U$, is of $D_{\alpha}$ $(0<\alpha<1)$ if and only if

$$
\sum_{n=0}^{\infty} n^{1-\alpha}\left|a_{n}\right|^{2}<\infty
$$

Received by the editors September 13, 1979 and, in revised form, February 7, 1980.

AMS (MOS) subject classifications (1970). Primary 30A74, 30A76. 
see, e.g., [4, pp. 8-9]. For the proof of $D_{1}=H^{2}$, see [9, Remark (a), p. 208]. Thus, $f \in D_{1}$ if and only if $\sum_{n=0}^{\infty}\left|a_{n}\right|^{2}<\infty$.

2. Proof of Theorem 1. Suppose that $\left\{z_{n}\right\}_{n=1}^{\infty}$ is a sequence of points in $U$, converging to 1 along the chord $X$ of the unit circle connecting 1 and $i$. Suppose further that

$$
\sum_{n=1}^{\infty}\left(\log \frac{1}{1-\left|z_{n}\right|^{2}}\right)^{-1}<\infty
$$

Then it follows from [7, Theorem 1, p. 220] (see also the last sentence in [7, p. 220]) that there exists a function $g \in D$ whose zeros are exactly 0 and $z_{n}, n=1,2, \ldots$. The function we wish to examine is the quotient $f=f_{1} / f_{2}$, where

$$
f_{1}(z)=(z-1) g(z) \text { and } f_{2}(z)=\overline{g(\bar{z})}, \quad z \in U .
$$

To prove that $f$ is a Dirichlet quotient we note first that $f_{2} \in D$. To show that $f_{1} \in D$ let $g(z)=\sum_{n=0}^{\infty} b_{n} z^{n}, z \in U$. Then

$$
\iint_{U}|g(z)|^{2} d x d y=\pi \sum_{n=0}^{\infty}(n+1)^{-1}\left|b_{n}\right|^{2}<\pi \sum_{n=0}^{\infty} n\left|b_{n}\right|^{2}<\infty .
$$

Since $\left|f_{1}^{\prime}\right|^{2}<2|g|^{2}+8\left|g^{\prime}\right|^{2}$, it follows that $f_{1} \in D$. To prove the nonnormality of $f$, we first note that $|f(z)|=|z-1| \rightarrow 0$ as $z \rightarrow 1$ along the radius $R=\{0<z<1\}$ of $U$ at 1 . Assume that $f$ is normal in $U$. It then follows from the result of Lehto and Virtanen [5, Theorem 2] that $f$ must possess zero as the angular limit at 1 . This is a contradiction because $f\left(\bar{z}_{n}\right)=\infty, n=1,2, \ldots$.

3. Proof of Theorem 2. We begin with a lemma on a Blaschke product

$$
B(z)=\prod_{n=1}^{\infty} \frac{\left|c_{n}\right|}{c_{n}} \frac{c_{n}-z}{1-\bar{c}_{n} z}, \quad z \in U,
$$

where $0<\left|c_{n}\right|<1(n=1,2, \ldots)$ and $\Sigma\left(1-\left|c_{n}\right|\right)<\infty$.

Lemma. Suppose that $\sum_{n=1}^{\infty}\left(1-\left|c_{n}\right|\right)^{\beta}<\infty$ for $0<\beta<1$. Then $B$ of (3.1) is $a$ member of $D_{\beta}$.

In effect, it follows from [6, Theorem 1] that

$$
\iint_{U}(1-|z|)^{\beta-1}\left|B^{\prime}(z)\right| d x d y<\infty .
$$

On the other hand, the Schwarz-Pick lemma yields

$$
(1-|z|)\left|B^{\prime}(z)\right|<\left(1-|z|^{2}\right)\left|B^{\prime}(z)\right|<1-|B(z)|^{2}<1,
$$

whence

$$
(1-|z|)^{\beta}\left|B^{\prime}(z)\right|^{2}<(1-|z|)^{\beta-1}\left|B^{\prime}(z)\right|, \quad z \in U .
$$

Combining (3.2) and (3.3) one observes that $B \in D_{\beta}$.

For the proof of Theorem 2, we first choose a sequence $\left\{a_{n}\right\}_{n=1}^{\infty}$ of points in $U$ converging to 1 along the chord $X$. We further suppose that $\left|a_{n}\right|=1-\gamma^{n}, n=1$, $2, \ldots$, where $0<\gamma<1$ is a constant. Then 


$$
\sum_{n=1}^{\infty}\left(1-\left|a_{n}\right|\right)^{\alpha}<\infty
$$

Next choose $\lambda>1 / \alpha$ and set $b_{n}=1-(n+1)^{-\lambda}, n=1,2, \ldots$ Consider the Blaschke products

$$
B_{3}(z)=\prod_{n=1}^{\infty} \frac{\left|a_{n}\right|}{a_{n}} \frac{a_{n}-z}{1-\bar{a}_{n} z} \quad \text { and } \quad B_{4}(z)=\prod_{n=1}^{\infty} \frac{b_{n}-z}{1-b_{n} z} .
$$

The Blaschke quotient we wish to examine is $Q=B_{1} / B_{2}$, where

$$
B_{1}(z)=B_{3}(z) B_{4}(z) \text { and } B_{2}(z)=\overline{B_{3}(\bar{z})}, \quad z \in U .
$$

Since $\sum_{n=1}^{\infty}\left(1-b_{n}\right)^{\alpha}<\infty$, it follows from the lemma, together with (3.4), that both $B_{1}$ and $B_{2}$ are of class $D_{\alpha}$. To prove the nonnormality of $Q$ we first prove that $B_{4}(z) \rightarrow 0$ as $z \rightarrow 1$ along the radius $R$. Denoting the non-Euclidean distance in $U$ by $\rho(\cdot, \cdot)$, we observe that the sequence

$$
\rho\left(b_{n}, b_{n+1}\right)=\frac{1}{2} \log \frac{1-b_{n} b_{n+1}+b_{n+1}-b_{n}}{1-b_{n} b_{n+1}-b_{n+1}+b_{n}}
$$

tends to zero as $n \rightarrow \infty$. It then follows from [1, Theorem 2] that the bounded function $B_{4}$ has the angular limit zero at 1 . By the same reasoning as in the last part of $\S 2, Q$ is not normal in $U$.

REMARK. Cima [2, Theorem 4] found a nonnormal Blaschke quotient

$$
\left(\prod_{n=1}^{\infty} \frac{\left|c_{n}\right|}{c_{n}} \frac{c_{n}-z}{1-\bar{c}_{n} z}\right) /\left(\prod_{n=1}^{\infty} \frac{\left|c_{n}^{\prime}\right|}{c_{n}^{\prime}} \frac{c_{n}^{\prime}-z}{1-\bar{c}_{n}^{\prime} z}\right)
$$

such that $\inf _{j, k>1} \rho\left(c_{j}, c_{k}^{\prime}\right)>0$; in other words, the distance between the zeros $\left\{c_{n}\right\}$ and the poles $\left\{c_{n}^{\prime}\right\}$ is positive. Our quotient $Q$ also satisfies this property. For, let $\bar{X}$ be the reflexion of $X$ with respect to the real axis. Then the non-Euclidean distance between $\bar{X}$ and the union $X \cup R$ is positive.

\section{REFERENCES}

1. F. Bagemihl and W. Seidel, Sequential and continuous limits of meromorphic functions, Ann. Acad. Sci. Fenn. Ser. A I 280 (1960), 1-17.

2. J. A. Cima, A nonnormal Blaschke-quotient, Pacific J. Math. 15 (1965), 767-773.

3. P. L. Duren, Theory of $\boldsymbol{H}^{p}$ spaces, Academic Press, New York and London, 1970.

4. V. I. Gavrilov, On the theorems of Beurling, Carleson and Tsuji on exceptional sets, Mat. Sb. 94 (130) (1974), 3-15; English transl. in Math. USSR Sb. 23 (1974), 1-12.

5. O. Lehto and K. I. Virtanen, Boundary behaviour and normal meromorphic functions, Acta Math. 97 (1957), 47-65.

6. D. Protas, Blaschke products with derivative in $H^{p}$ and $B^{p}$, Michigan Math. J. 20 (1973), 393 - 396.

7. H. S. Shapiro and A. L. Shields, On the zeros of functions with finite Dirichlet integral and some related function spaces, Math. Z. 80 (1962), 217-229.

8. N. Yanagihara, On a quotient of functions with finite Dirichlet integrals, J. College Arts Sci. Chiba Univ. 4 (1966), no. 4, 395.

9. A. Zygmund, Trigonometric series. II, Cambridge Univ. Press, London and New York, 1959.

Department of Mathematics, Tokyo Metropolitan University, Fukazawa, Setagaya-ku, TOKYO 158, JAPAN 